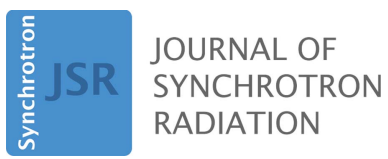

ISSN 1600-5775

Received 4 August 2020

Accepted 10 December 2020

Edited by S. M. Heald, Argonne National Laboratory, USA

Keywords: bidirectional scanning methods; STXM; S-track; post-processing; SSRF.

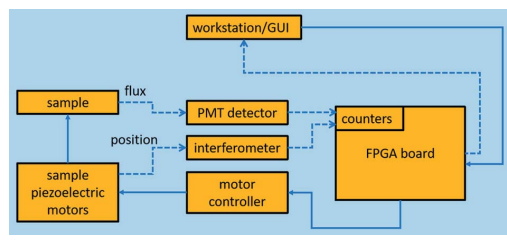

C 2021 International Union of Crystallography

\section{A bidirectional scanning method for scanning transmission X-ray microscopy}

\author{
Tianxiao Sun, ${ }^{\mathrm{a}, \mathrm{b}}$ Xiangzhi Zhang, ${ }^{\mathrm{a}, \mathrm{c} *}$ Zijian $\mathrm{Xu},{ }^{\mathrm{a}, \mathrm{c} *}$ Yong Wang, ${ }^{\mathrm{a}, \mathrm{c}}$ Zhi Guo, ${ }^{\mathrm{a}, \mathrm{c}}$ \\ Jian Wang ${ }^{\mathrm{d}}$ and Renzhong Tai ${ }^{\mathrm{a}, \mathrm{c} *}$
}

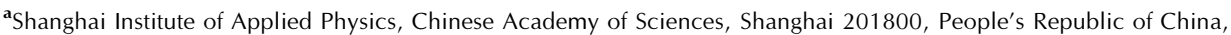
${ }^{\mathbf{b}}$ University of Chinese Academy of Sciences, Beijing 100049, People's Republic of China, 'Shanghai Synchrotron Radiation Facility, Shanghai Advanced Research Institute, Chinese Academy of Sciences, Shanghai 201210, People's Republic of China, and ${ }^{\mathbf{d}}$ Canadian Light Source Inc., University of Saskatchewan, 44 Innovation Boulevard, Saskatoon, Saskatchewan S7N 2V3, Canada. *Correspondence e-mail: zhangxiangzhi@zjlab.org.cn, xuzijian@zjlab.org.cn, tairenzhong@sinap.ac.cn
\end{abstract}

Scanning mode is a key factor for the comprehensive performance, including imaging efficiency, of scanning transmission X-ray microscopy (STXM). Herein is presented a bidirectional scanning method designed for STXM with an S-shaped moving track. In this method, artificially designed ramp waves are generated by a piezo-stage controller to control the two-dimensional scanning of the sample. The sample position information is measured using laser interferometric sensors and sent to a field-programmable gate array (FPGA) board which also acquires the X-ray signals simultaneously from the detector. Since the data recorded by the FPGA contain the real position of each scanned point, the influence of the backlash caused by the back-turning movement on the STXM image can be eliminated. By employing an adapted post-processing program, a re-meshed high-resolution STXM image can be obtained. This S-track bidirectional scanning method in fly-scan mode has been implemented on the STXM endstation at the Shanghai Synchrotron Radiation Facility (SSRF), and successfully resolved the $\sim 30 \mathrm{~nm}$ interval between the innermost strips of a Siemens star. This work removes the limitation on bidirectional scanning caused by motor backlash and vibration, and significantly improves the efficiency of STXM experiments.

\section{Introduction}

Scanning transmission X-ray microscopy (STXM) is a synchrotron-based X-ray imaging technique under rapid development (Takeichi et al., 2016; Lühl et al., 2019; Witte et al., 2020). The technique combines nanoscale imaging with spectral sensitivity (Kaznatcheev et al., 2007; Ma et al., 2019), and thus becomes a powerful tool to probe the properties of solids (Nilsson et al., 2005; Zou et al., 2006; Hub et al., 2010), liquids (Smith et al., 1998; Beetz \& Jacobsen, 2003; Wang et al., 2011), films (Lee et al., 2013; Ling et al., 2014; Zhang et al., 2019), particles (Wan et al., 2007; Bahadur et al., 2010; Remusat et $a l ., 2012$ ) and other forms of specimen in the materials, life, energy and environmental sciences at the sub-micrometre scale. However, since a high-resolution image needs to scan a lot of pixels, the scanning speed is critical for STXM (Kilcoyne et al., 2003; Zobelli et al., 2019). Quick scanning could not only greatly improve the efficiency of experiments, but also reduce sample damage caused by X-ray dose accumulation (Obst et al., 2009). Currently, the fly-scan method greatly reduces the time required for STXM imaging, which enables STXM-based nanotomography and energy-stack imaging (Leontowich et al., 2018). However, with the continuous development of science and technology, the increasing needs of users and emerging 

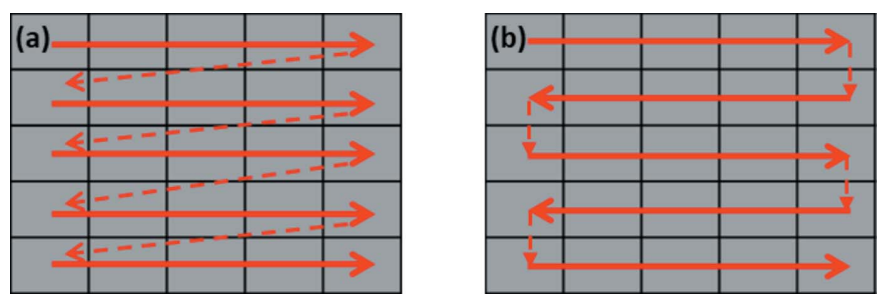

Figure 1

(a) A schematic diagram of a unidirectional scan pattern. (b) A schematic diagram of a bidirectional scan pattern. For the same scanning range, the bidirectional scanning path is shorter than the unidirectional one, which can greatly increase the scanning efficiency.

complex imaging methods are stimulating the creation of faster scanning technologies for higher-efficiency STXM imaging.

In STXM, the X-ray beam is typically scanned across the region of interest following a sawtooth path (Schmahl et al., 1995; Kaznatcheev et al., 2007; Takeichi et al., 2016; Leontowich et al., 2018), as Fig. 1(a) shows, and this is called the unidirectional scan pattern. In this traditional scan method, the precise scan pattern is defined before the acquisition, and then a full image matrix is recorded in accordance with the pixel scanning order (Takeichi et al., 2016). This method spends approximately a quarter of the total imaging time on return trips. A bidirectional scanning method which can realize round-trip scanning path can effectively reduce the redundancy. Image quality problems (noisy images, artifacts, poor spatial resolution etc.) caused by backlash error and vibration have limited the application of bidirectional scanning in the past.

In this work, an efficient bidirectional scanning method in fly-scan mode with an S-shaped moving track was designed for STXM via a field-programmable gate array (FPGA) board. A piezo-stage controller generates designed ramp waves to control the two-dimensional movement of the sample. By using a laser interferometer, the real sample positions can be measured. Furthermore, the FPGA board can collect the sample position information and the detector signal simultaneously. Since the data recorded by the FPGA contain the real position of each scanned point, the backlash error caused by the turning-back movement can be removed. By employing an adapted post-processing program, a re-meshed high-resolution STXM image can be obtained from the raw data. So far the S-track bidirectional scanning method has been implemented on the STXM endstation at the Shanghai Synchrotron Radiation Facility (SSRF). This work eliminates the limitation on bidirectional scanning caused by motor backlash and vibration, and significantly improves the efficiency of STXM experiments.

\section{The conception of the bidirectional scanning method}

In order to better understand the new scanning method, the schematics of the common unidirectional scan pattern and the S-track bidirectional scan pattern are shown in Figs. 1(a) and $1(b)$, respectively. Unidirectional scanning is a traditional
Z-type scanning to avoid backlash error. After scanning one horizontal line, the motor returns to the starting point of the next line and starts again (Huang et al., 2015; Leontowich et al., 2018). In this method, the detected X-ray flux signals are arranged directly in time order to form an image. This usually requires a high stability of the system to ensure that the actual scanning path has little deviation from the expected one (Huang et al., 2015; Roy et al., 2019). This high-precision requirement sets an upper limit on the motor speed. In addition, the redundancy of the motor travel length greatly increases the time required for STXM imaging.

A more radical conceptual leap is that the backlash does not need to be worried about at all in S-track scanning. All that is needed is that the X-ray probe travels across every position on the sample, whether or not there is any backlash or deviation relative to the expected path. Thus, the only requirement is that the absolute position of the sample be recorded in real time while recording the X-ray signals. Once these data have been measured, the X-ray signals can be remapped onto their real positions, so that the STXM image can be obtained computationally. This method allows the sample to be scanned bidirectionally at a faster speed. Even if backlash and motor vibration are present, a high-quality scanned image can still be obtained by rearranging a scattered distribution of the absolute positions of all pixel signals into a regular mesh grid. Compared with unidirectional scanning, this novel S-track scanning has a shorter travel path, which results in higher experimental efficiency.

\section{Practical implementation in STXM}

The advancement of the scanning control modules for STXM now allows us to generate arbitrary scanning patterns and exploit new illumination modes (Wojdyla et al., 2016). The bidirectional scanning method proposed here was implemented based on a customized FPGA board (XC7Z0202CLG400I, Xilinx, USA) with $100 \mathrm{MHz}$ clock frequency. Fig. 2 shows the practical implementation logic for bidirectional scanning. The FPGA board is the core of the entire system and responsible for the strict synchronization of the scan module with the data acquisition module.

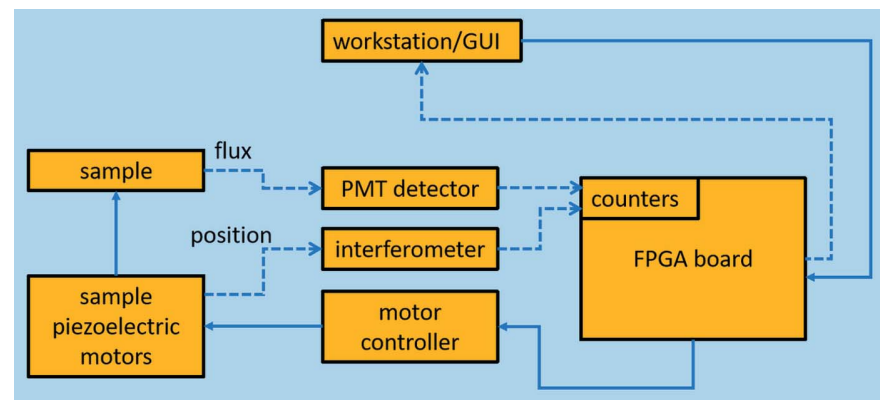

Figure 2

A flow chart of the bidirectional scanning method controls. Dotted arrows indicate information transfer directions and solid arrows indicate the control relationships. 
A head-on type photomultiplier tube (PMT) (R647P, Hamamatsu Photonics, Japan) with a photon-counting unit (C9744, Hamamatsu Photonics, Japan) and a laser interferometer (IDS3010, attocube systems AG, Germany) act as detectors for data acquisition. They send the obtained X-ray signals and sample positions to the FPGA board continuously. The FPGA board is equipped with a multi-channel counter module which can synchronously collect the incoming X-ray signals and position information at a fixed frequency up to $100 \mathrm{MHz}$. These signals are then packaged and sent to a workstation that has been installed with a home-developed GUI program named STXM-daq. The Python-based STXM-daq integrates motion control, data display, data postprocessing and other user interactions (such as X-ray energy change, imaging mode selection, raster switching etc.). Through the post-process module of this program, an interpolation mathematics model can be used to rearrange the incoming X-ray signals according to the real location information, and display the refined STXM image to the user in real time.

The implementation of the new scan module relies on a wave generator installed in the motor controller [E-712, Physik Instrumente (PI) GmbH, Germany] which can control/ drive each axis motor according to user-specified patterns, the so-called 'waveforms', which are set by users in STXM-daq. The system can automatically generate these waveforms and store them in the volatile memory of the scan module, waiting for a trigger signal to output and drive the motors.

Due to temperature, vibration, friction and other external factors, the actual motion track of the sample motor in highspeed motion may be inconsistent with the ideal output waveform. Although this bidirectional scanning method can eliminate a certain degree of trajectory deviation by recording the real position of the sample in real time, a trajectory less affected by noise will still make the signal more uniform and the calibrated image obtained later will be more accurate. In order to alleviate this problem, the PID (proportional integral differential) algorithm is used to calibrate the movement deviation. The PID control algorithm comes from the built-in control algorithm module of the PI E-712 controller, which can be activated by the relevant PI General Command Set (GCS). In a closed-loop operation for this positioning system with a horizontal piezo-motor (P-621.1CD, PI) and a vertical piezo-motor (P-621.ZCD, PI), the main component of the tracking error is the phase shift between the specified position and the real position. By adding a feedforward signal to the control algorithm this phase shift can be reduced. Moreover, for bidirectional STXM imaging, the tracking error is not a constant like other types of linear PID servo system, but changes with the scanning position and direction. This is due to the nonlinearity of the piezo motor and the limited dynamic performance of the servo controller. As a result, the scanning position is not directly proportional to time but shows dynamic nonlinearity.

Considering the particularity of the bidirectional scanning periodic motion, a better way of reducing tracking error is to monitor the errors for an axis during one or more periods and then compensate for them in all subsequent periods. Thus, another PI E-712 built-in control algorithm, Dynamic Digital Linearization $(D D L)$, is employed to achieve significantly better position accuracy in dynamic applications of STXM imaging with periodic motions. The dedicated $D D L$ algorithm records the motion of the motor over one or more wavegenerator output cycles. The information collected is written to the internal memory of the controller and can then be used to refine the output control signals. After several lines of scanning, the tracking error will be greatly reduced (Leontowich et al., 2018).

Fig. 3(a) shows predefined waveforms of the horizontal and vertical piezoelectric motors for the sample. This diagram implies that the motor in the horizontal direction (blue line) will move repeatedly back and forth in the range of 0 to $100 \mu \mathrm{m}$ at a fixed speed, while the motor in the vertical direction (orange line) moves from 0 to $100 \mu \mathrm{m}$ within $30 \mathrm{~s}$ at a certain frequency. This programmable motor control mechanism facilitates the synchronization of movement in the two directions. Furthermore, the high speed and concurrency
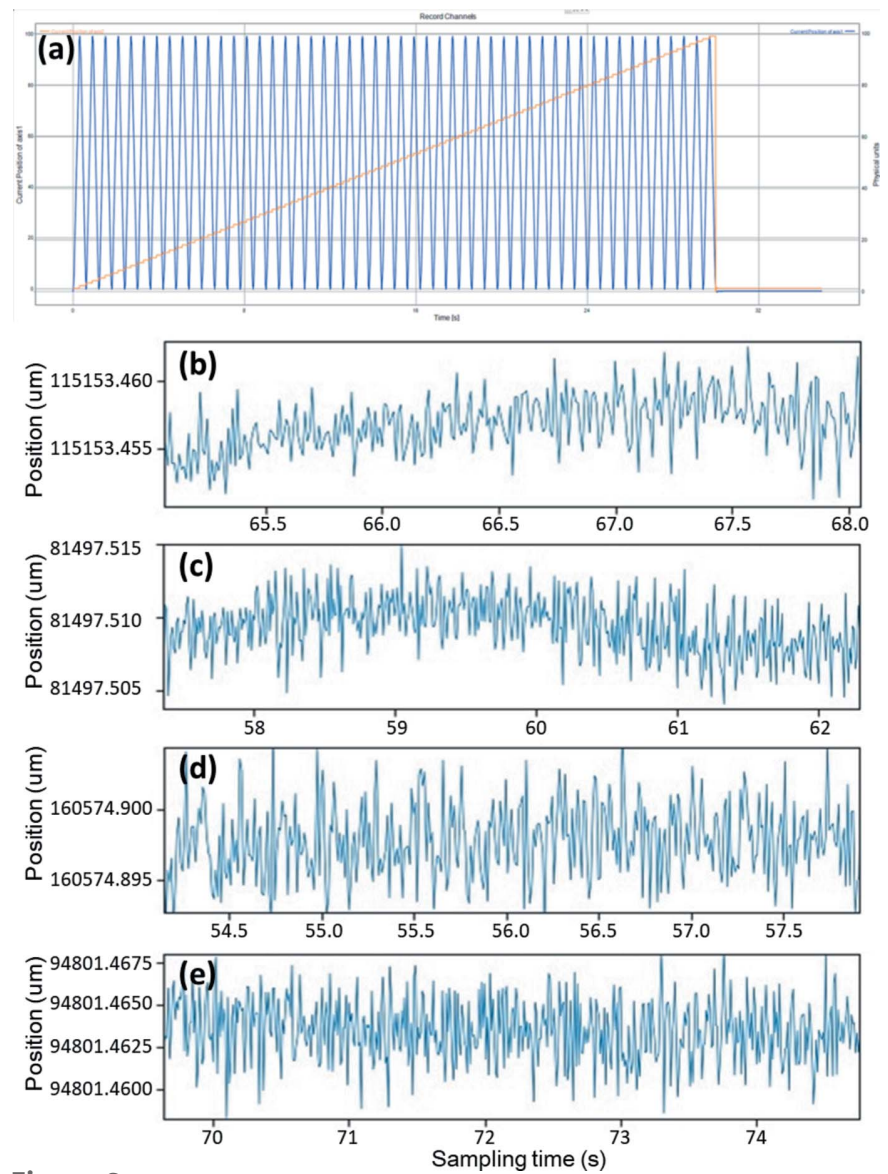

Figure 3

(a) A plot of the driving waveforms of the piezoelectric motors in the two directions of sample scanning. The orange line represents the control of the vertical motor and the blue line represents the control of the horizontal motor. It will take $30 \mathrm{~s}$ to scan $100 \times 100$ pixels with a dwell time of $3 \mathrm{~ms}$, while the FPGA reads data ten times per dwell time. (b) Vibration test chart of the zone plate in the $X$ direction. (c) Vibration test chart of the zone plate in the $Y$ direction. (d) Vibration test chart of the sample in the $X$ direction. (e) Vibration test chart of the sample in the $Y$ direction. 
of the FPGA ensure that the data acquisition and sample movement are triggered simultaneously. Figs. $3(b)$ to $3(e)$ show the vibration levels of the zone plate $X$, zone plate $Y$, sample $X$ and sample $Y$, respectively, in the non-scanning state. Taken together, the vibration amplitude of the system is only $5-10 \mathrm{~nm}$ in a short period of time.

Because the real sample position for each pixel of the raw STXM image is recorded by the FPGA in real time, an accurate output image can be recovered through rearranging all pixel signals from the scattered/fluctuating absolute positions into a regular rectangular mesh grid with an interpolation mathamatics approach. Fig. $4(a)$ is a schematic diagram of rearranging the pixel signals. This post-processing can effectively eliminate the impact of motor backlash and vibration during bidirectional scanning. The STXM-daq software incorporates this image-recovery module to remove image distortion, unevenness and other artifacts originating from the physical experimental environment. Fig. 4(b) is an algorithm flowchart of the image-recovery function module. In this module, a standard rectangular mesh grid is automatically created according to the pixel number of the raw image and then filled with modified X-ray signals based on an average over the raw signals falling into respective grid points.

\section{Experimental results and post-processing}

Bidirectional scanning test experiments were carried out on the newly built STXM endstation on beamline BL08U1A at the SSRF, and the unidirectional scanning experiments for comparison were conducted on the ambient STXM instrument on the SM beamline at the Canadian Light Source (CLS). Fig. 5(a) shows a raw STXM image of a Siemens star measured through the bidirectional scanning method at $760 \mathrm{eV}$. The image has $1000 \times 1000$ pixels and covers a $10 \mu \mathrm{m} \times 10 \mu \mathrm{m}$ area. The dwell time is $1 \mathrm{~ms}$ for data collection, and to improve information richness the $\mathrm{X}$-ray signals and position information are recorded ten times within each dwell time. The image data acquisition took $16.6 \mathrm{~min}$. The raw image [Fig. 5(a)] is a direct mapping of the $\mathrm{X}$-ray signals according to their position information recorded by the interferometer, showing a very poor image quality. We can see many sawtooth or jagged structures on both sides of the stripes, which result from the

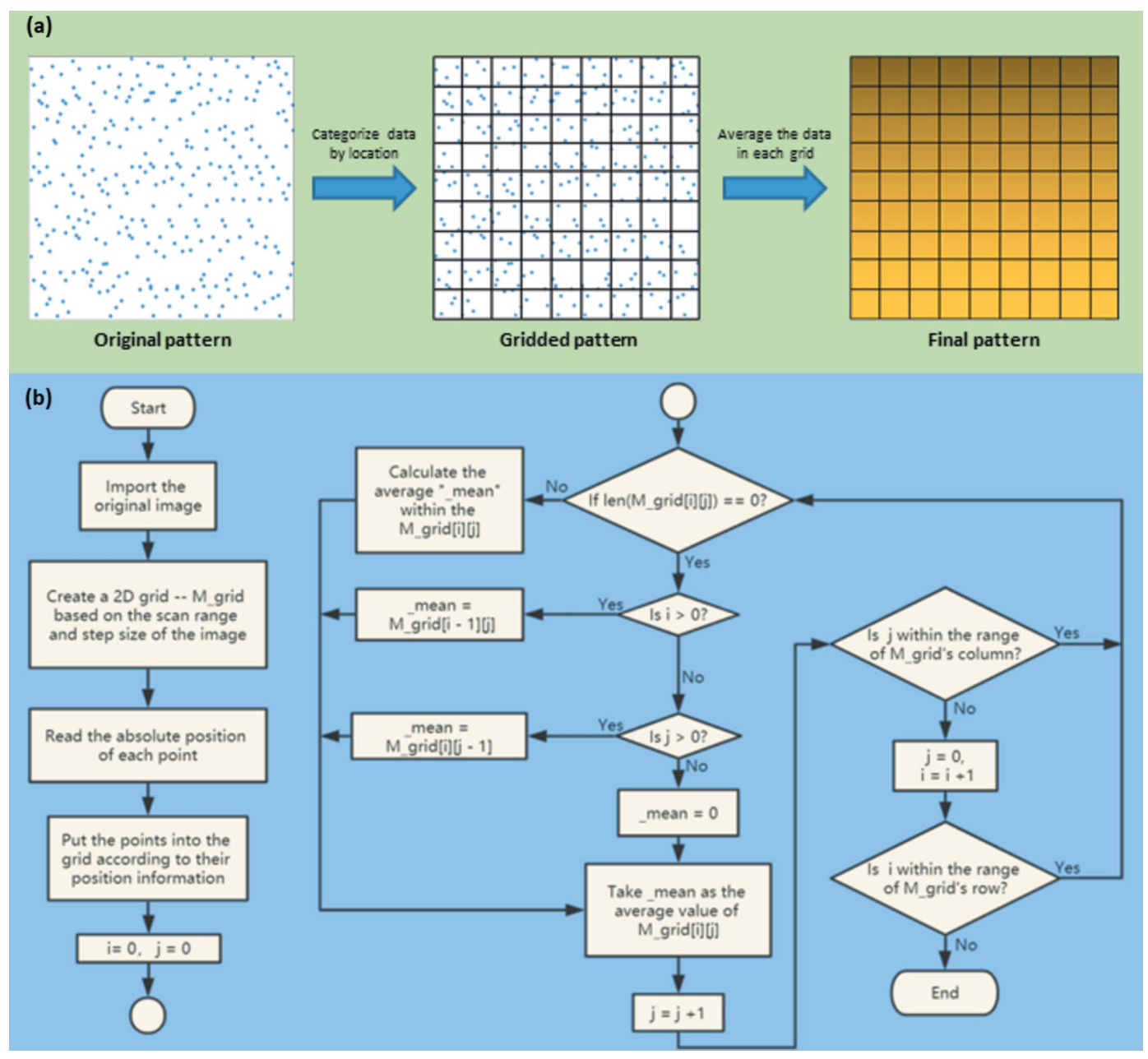

Figure 4

Data post-processing. (a) A schematic diagram of pixel rearranging. (b) A flow chart of the image-recovery algorithm embedded in the STXM-daq software. This module re-meshes the X-ray signals from a scattered position distribution into a regular grid by averaging over the raw data falling in each new grid point. 


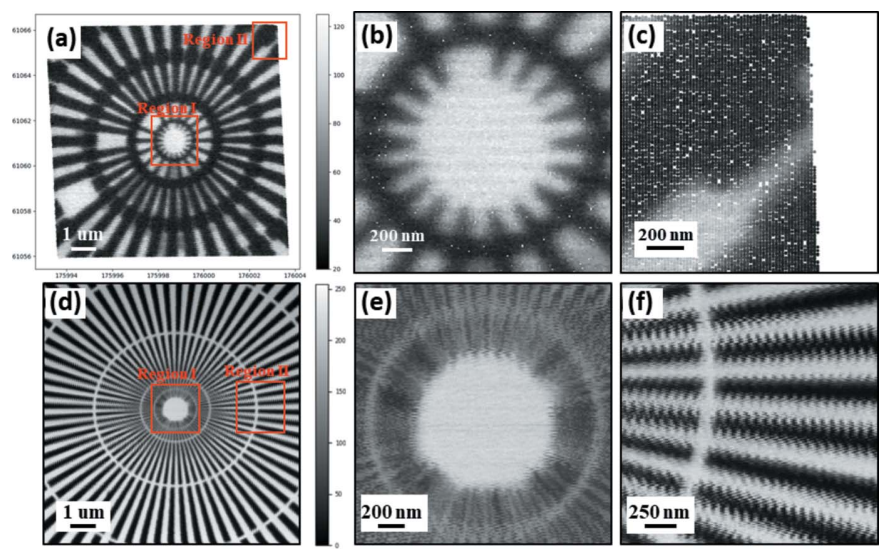

Figure 5

Comparison of bidirectional scanning and traditional unidirectional scanning results. (a) A high-resolution Siemens star pattern measured by the bidirectional scanning method at $760 \mathrm{eV}$, with a $10 \mu \mathrm{m} \times 10 \mu \mathrm{m}$ range, $1000 \times 1000$ pixels and $1 \mathrm{~ms}$ dwell time. (b) An enlarged view of region I in panel $(a)$. (c) An enlarged view of region II in panel $(a)$. (d) Under the same scan parameters, the ANT star pattern measured by the traditional unidirectional scanning method at the Canadian Light Source. $(e)$ An enlarged view of region I in panel $(d)$. $(f)$ An enlarged view of region II in panel $(d)$.

backlash of the horizontal motor. In addition, the image is entirely distorted or tilted, with the circles transformed into ellipses, which could be caused by the inclination of the reflection mirrors for the laser interferometer. Fig. $5(b)$ is an enlarged view of a central part of the image [Fig. 5(a), region I], illustrating missing pixels caused by motor vibration. Fortunately, images acquired by bidirectional scanning will not be misaligned or distorted due to the vibration of the scanning system or external influences. Fig. 5(c) shows the backlash effects caused by the turning-back motion of the motor, which lead to the jagged edges of the image [Fig. 5(a), region II]. Fig. 5(d) is a unidirectional scanning image of a star pattern under the same scan parameters at the CLS. This image was also taken in fly-scan mode and took $34.7 \mathrm{~min}$. Fig. 5(e) is an enlarged view of region I in $5(d)$. The tip of the $\sim 25 \mathrm{~nm}$ innermost ring is relatively fuzzy. Meanwhile, external interference or vibration of the scanning system causes a relatively large periodic misalignment of the image measured by unidirectional scanning, which will cause the image to differ greatly from the actual morphology of the sample.

After post-processing of the raw image by rearranging and modifying all pixel X-ray signals from the scattered absolute positions into a regular mesh grid with an interpolation mathematics approach embedded in the STXM GUI software, a precise high-resolution reconstructed image was obtained, as Fig. 6(a) shows. For the processed STXM image, we used a mesh with $625 \times 625$ grid points to re-mesh the original data, so the final STXM pixel size is $16 \mathrm{~nm}$. Through the reconstruction algorithm for the image, the pixel distribution becomes completely uniform and all the missed pixels are filled up, as the enlarged image [Fig. 6(b)] shows. From Figs. $6(a)-6(c)$, the sawtooth or jagged structures at the edges of many strips of the raw image disappear completely. In addition, the tilted image edge in Fig. 6(c) also becomes much
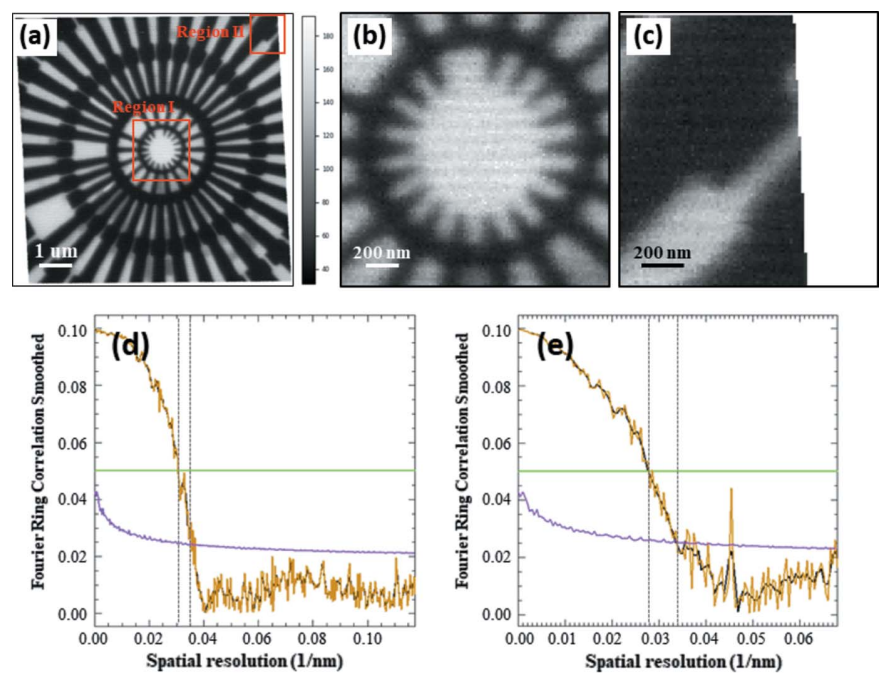

Figure 6

The final output image of the bidirectional scanning method after the remeshing process, and comparison between the resolution of bidirectional versus unidirectional scanning. (a) The final refined picture after the image reconstruction processing. (b) The internal pixel arrangement for region I in panel $(a)$, showing a uniform distribution with no missed pixels due to motor vibration. (c) The jagged structures in region II in Fig. 5(a) caused by motor backlash are also eliminated and the edge becomes smoother. (d) Resolution analysis of the traditional unidirectional scanning image [Fig. $5(d)$ ] using the Fourier ring correlation (FRC) method. (e) Resolution analysis of the post-processed bidirectional scanning image [Fig. 6(a)] using the FRC method.

smoother than that in Fig. 5. It can be seen that the innermost ring tip of the Siemens star, with a width of $30 \mathrm{~nm}$, can be basically distinguished.

In order to quantify the spatial resolution of the result, several methods were used. Fig. 6(e) presents the calculated Fourier ring correlation (FRC) (Shapiro et al., 2014; Wu et al., 2018; Koho et al., 2019) of the post-processed bidirectional scanning image in Fig. 6(a). The FRC curves of the final output image of the bidirectional scanning method [brown and black lines in Fig. 6(e)] drop below the chosen threshold line of 0.5 and the half-bit threshold at spatial frequencies of 0.0279 and $0.0340 \mathrm{~nm}^{-1}$, respectively, which correspond to spatial resolutions of 35.8 and $29.4 \mathrm{~nm}$, respectively. For comparison, the resolutions of the conventional unidirectional scanning image [Fig. 5(d)] can be calculated as 33.2 and $26.7 \mathrm{~nm}$ in Fig. 6(d). It can be seen that bidirectional scanning can effectively achieve the same level of resolution as unidirectional scanning.

In addition, efficient bidirectional scanning will bring the advantage of a low radiation dose. The X-ray imaging dose $D$ is often estimated from the total interaction cross section (Jones et al., 2017; Beetz \& Jacobsen, 2003):

$$
D=\left(I_{0} E t \mu / \rho A \eta\right) \text { Gy, }
$$

where $I_{0}$ is the incident photon intensity, $E$ is the photon energy in electronvolts, $\eta$ is the efficiency of the detector, $t$ is the exposure time in milliseconds, $\rho$ is the density in $\mathrm{g} \mathrm{cm}^{3}, A$ is the irradiated area in $\mu \mathrm{m}^{2}$ and $\mu$ is the inverse absorption length in $\mu \mathrm{m}^{-1}$. For Figs. 5(a) and 5(d), if we regard conditions such as the incident photon intensity, the irradiated area of the samples and their material as the same, then the only differ- 
ence between the two images is the illumination time. The time taken for bidirectional scanning is 0.478 times that for unidirectional scanning. Thus, according to equation (1), the radiation dose for bidirectional scanning is also 0.478 times that for unidirectional scanning.

\section{Summary and perspectives}

In this paper, we have presented a bidirectional scanning implementation method for STXM with an S-shaped moving track. This scanning imaging scheme was tested on the STXM beamline at the SSRF and a $1000 \times 1000$ pixel high-quality output image with a resolution of about $30 \mathrm{~nm}$ to $50 \mathrm{~nm}$ could be obtained within $16.6 \mathrm{~min}$.

Through the bidirectional scanning S-track motion controlled by an FPGA and its supporting image-recovery software, a new generation of STXM with a high resolution and faster data acquisition was successfully implemented. It can remove or relieve restrictions imposed by motor backlash and vibrations and the instability of the STXM system, without degradation of the image quality. The scanning method has a shorter scanning path than traditional unidirectional scanning, which makes imaging more efficient and lowers the radiation dose on the sample.

The method is currently unable to perform image reconstruction in real time in experiments due to the slow postprocessing speed. It will be further improved and perfected to make the processing of the raw data more efficient by optimizing and accelerating related STXM-daq modules, thus enhancing the user's experience of real-time STXM imaging. In addition, this method will be applied to time-consuming imaging methods such as nano-CT and energy stack imaging, so that their imaging time can be reduced markedly.

\section{Acknowledgements}

We thank the BL08U1A beamline of the Shanghai Synchrotron Radiation Facility for providing beamtime to test the new scanning method, and thank Tolek Tyliszczak for his helpful suggestions and for sharing useful experience for the SSRF STXM upgrade. We also thank the Canadian Light Source for providing beam time and guiding information for the SSRF STXM upgrade.

\section{Funding information}

The following funding is acknowledged: National Natural Science Foundation of China (grant Nos U1832154, 11875316 and U1832146).

\section{References}

Bahadur, R., Russell, L. M. \& Prather, K. (2010). Aerosol Sci. Technol. 44, 551-562.

Beetz, T. \& Jacobsen, C. (2003). J. Synchrotron Rad. 10, 280-283.

Huang, X., Lauer, K., Clark, J. N., Xu, W., Nazaretski, E., Harder, R., Robinson, L. K. \& Chu, Y. S. (2015). Sci. Rep. 5, 9074.
Hub, C., Burkhardt, M., Halik, M., Tzvetkov, G. \& Fink, R. (2010). J. Mater. Chem. 20, 4884-4887.

Jones, M. W. M., Hare, D. J., James, S. A., de Jonge, M. D. \& McColl, G. (2017). Anal. Chem. 89, 12168-12175.

Kaznatcheev, K. V., Karunakaran, C., Lanke, U. D., Urquhart, S. G., Obst, M. \& Hitchcock, A. P. (2007). Nucl. Instrum. Methods Phys. Res. A, 582, 96-99.

Kilcoyne, A. L. D., Tyliszczak, T., Steele, W. F., Fakra, S., Hitchcock, P., Franck, K., Anderson, E., Harteneck, B., Rightor, E. G., Mitchell, G. E., Hitchcock, A. P., Yang, L., Warwick, T. \& Ade, H. (2003). J. Synchrotron Rad. 10, 125-136.

Koho, S., Tortarolo, G., Castello, M., Deguchi, T., Diaspro, A. \& Vicidomini, G. (2019). Nat. Commun. 10, 3103.

Lee, V., Susac, D., Kundu, S., Berejnov, V., Atanasoski, R. T., Hitchcock, A. P. \& Stumper, J. (2013). ECS Trans. 58(1), 473-480.

Leontowich, A. F. G., Berg, R., Regier, C. N., Taylor, D. M., Wang, J., Beauregard, D., Geilhufe, J., Swirsky, J., Wu, J., Karunakaran, C., Hitchcock, A. P. \& Urquhart, S. G. (2018). Rev. Sci. Instrum. 89, 093704.

Ling, S., Qi, Z., Watts, B., Shao, Z. \& Chen, X. (2014). Phys. Chem. Chem. Phys. 16, 7741-7748.

Lühl, L., Andrianov, K., Dierks, H., Haidl, A., Dehlinger, A., Heine, M., Heeren, J., Nisius, T., Wilhein, T. \& Kanngießer, B. (2019). J. Synchrotron Rad. 26, 430-438.

Ma, L., Zhang, X., Xu, Z., späth, A., Xing, Z., Sun, T. \& Tai, R. (2019). Opt. Express, 27, 7787-7802.

Nilsson, H. J., Tyliszczak, T., Wilson, R. E., Werme, L. \& Shuh, D. K. (2005). Anal. Bioanal. Chem. 383, 41-47.

Obst, M., Wang, J. \& Hitchcock, A. P. (2009). J. Phys. Conf. Ser. 186, 012045 .

Remusat, L., Hatton, P. J., Nico, P. S., Zeller, B., Kleber, M. \& Derrien, D. (2012). Environ. Sci. Technol. 46, 3943-3949.

Roy, K., Raabe, J., Schifferle, P., Finizio, S., Kleibert, A., van Bokhoven, J. A. \& Artiglia, L. (2019). J. Synchrotron Rad. 26, 785792.

Schmahl, G., Rudolph, D., Guttmann, P., Schneider, G., Thieme, J. \& Niemann, B. (1995). Rev. Sci. Instrum. 66, 1282-1286.

Shapiro, D. A., Yu, Y., Tyliszczak, T., Cabana, J., Celestre, R., Chao, W., Kaznatcheev, K., Kilcoyne, A. L. D., Maia, F., Marchesini, S., Meng, Y. S., Warwick, T., Yang, L. L. \& Padmore, H. A. (2014). Nat. Photon. 8, 765-769.

Smith, A. P., Bai, C., Ade, H., Spontak, R. J., Balik, C. M. \& Koch, C. C. (1998). Macromol. Rapid Commun. 19, 557-561.

Takeichi, Y., Inami, N., Suga, H., Miyamoto, C., Ueno, T., Mase, K., Takahashi, Y. \& Ono, K. (2016). Rev. Sci. Instrum. 87, 013704.

Wan, J., Tyliszczak, T. \& Tokunaga, T. K. (2007). Geochim. Cosmochim. Acta, 71, 5439-5449.

Wang, J., Zhou, J., Fang, H., Sham, T. K., Karunakaran, C., Lu, Y., Cooper, G. \& Hitchcock, A. P. (2011). J. Electron Spectrosc. Relat. Phenom. 184, 296-300.

Witte, K., Späth, A., Finizio, S., Donnelly, C., Watts, B., Sarafimov, B., Odstrcil, M., Guizar-Sicairos, M., Holler, M., Fink, R. H. \& Raabe, J. (2020). Nano Lett. 20, 1305-1314.

Wojdyla, J. A., Panepucci, E., Martiel, I., Ebner, S., Huang, C.-Y., Caffrey, M., Bunk, O. \& Wang, M. (2016). J. Appl. Cryst. 49, 944952.

Wu, J., Zhu, X., West, M. M., Tyliszczak, T., Shiu, H., Shapiro, D. A., Berejnov, V., Susac, D., Stumper, J. \& Hitchcock, A. P. (2018). J. Phys. Chem. C, 122, 11709-11719.

Zhang, W., Melo, L. G. A., Hitchcock, A. P. \& Bassim, N. (2019). Micron, 120, 74-79.

Zobelli, A., Woo, S. Y., Tararan, A., Tizei, L. H., Brun, N., Li, X., Stéphan, O., Kociak, M. \& Tencé, M. (2019). Ultramicroscopy, 212, 112912.

Zou, Y., Araki, T., Appel, G., Kilcoyne, A. L. D. \& Ade, H. (2006). Chem. Phys. Lett. 430, 287-292. 